\title{
SOBRE EL CUADRANTE SOLAR DE ALFONSO X EL SABIO
}

\author{
Carlos Dorce Polo \\ Depto. de Filología Arabe - Facultad de Filología - Gran Via 585 - 08007 Barcelona
}

\section{RESUMEN}

Este artículo es un resumen del contenido del tratado alfonsí Libro del Relogio dicho de la piedra de la sombra (s. XIII), en el que su autor, Rabiçag, describe la construcción de un cuadrante solar horizontal. Este reloj del sol es básicamente el mismo que el descrito por el Battānī en sus Canones (s. IX) aunque presenta algunas innovaciones y comprobaciones que no están en el tratado oriental.

\section{SUMMARY}

Thsi article is a summary of the contents of the Alfonsine treatise called Libro del relogio dicho de la piedra de la sombra (13 ${ }^{\text {th }}$ c.), in which its author, Rabiçag, describes the construction of a sundial. This instrument is basically the same one described by al-Battāni $\left(9^{\text {th }} \mathrm{c}\right.$.) in his Canons, with the exeption that the Alfonsine text contains some innovations and proofs which are not to be found in the Oriental treatise.

Si dejamos de lado el tratado de Gnomónica de Ibn al-Raqqām (m. 1315), la historia de los cuadrantes solares de tradición andalusí se fundamenta en tres tipos de relojes solares, uno solo de los cuales aparece documentado arqueológicamente, mientras que de los otros dos sólo tenemos descripciones:

1) El reloj llamado balāta (figura 1) [CABANELAS, 1958; SAMSÓ, 1992; CASULLERAS, 1993; CASULLERÁs, 1996] documentado en al-Andalus desde el siglo X y del que aparece una descripción en el manuscrito de Ripoll 225. La balāta consiste en una piedra rectangular en la que en dos de sus ángulos hay colocados dos estiletes en los que convergen seis posibles radios de circunferencia separados entre sí por unos $15^{\circ}$. Uno de los dos grupos de seis líneas sirve para marcar las horas anteriores al mediodía y el otro, para las posteriores. Este tipo de instrumento, según J. Casulleras podría ser una deformación de un instrumento semejante al cuadrante ecuatorial de tradición greco-romana [CASULLERAS, 1996]. 


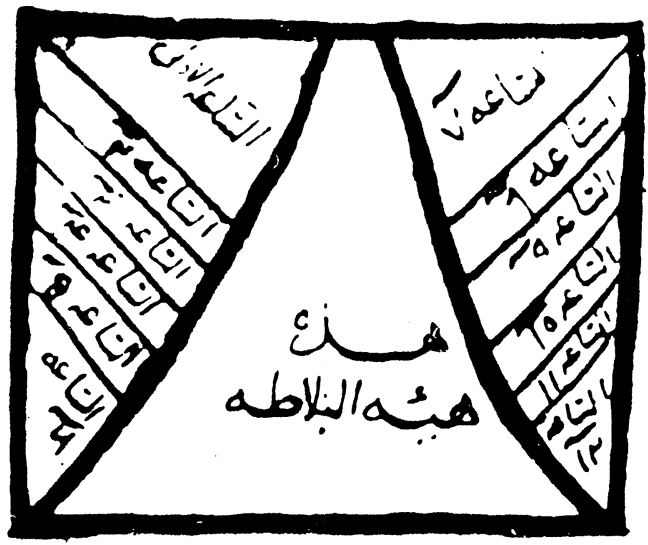

Figura 1. Balāta que aparece en el manuscrito de Carullah (Istambul) 1279.

2) Los cuadrantes cónicos y su variante cilíndrica citados por Azarquiel (m. 1100) en el prólogo a su tratado sobre la azafea [SAMSÓ, 1992]. En el Kitāb al-anwä' de alUmawī al-Qurtubī (m. 1206), el autor nos menciona un reloj de sol cónico llamado mukhula. El cuadrante cilíndrico, introducido en el mundo cristiano en el siglo XI, perduraba todavía a principios de siglo entre algunos pastores del Pirineo.

3) Finalmente, mencionar el reloj conocido como cuadrante solar horizontal. Conservamos fragmentos o piezas enteras de ocho cuadrantes solares horizontales [CABANELAS, 1958; KING, 1978; BARCELÓ, LABARTA, 1988; KING,1992; LABARTA, BARCELÓ, 1995]. Existen, por otra parte, textos que describen la construcción y uso de este tipo de cuadrantes. Éstos son básicamente dos: la Risāla $\mathrm{fi}^{c}{ }^{c}$ ilm al-zilāl de Ibn al-Raqqām al-Andalusì (s. XIV), editada, traducida y comentada por Joan Carandell en 1988. Este tratado, que no contiene ningún tipo de tablas, explica la construcción de varios tipos de relojes de sol entre los que se encuentra un cuadrante horizontal trazado mediante el método griego del analema. El segundo texto es el Libro del Relogio dicho de la piedra de la sombra (s. XIII) de los Libros del Saber de Alfonso $\mathrm{X}$ el Sabio [RICO, IV, pp. 3-23] en el que, como se va a ver inmediatamente, se describe un reloj de sol horizontal que es básicamente el recogido por al-Battāni en sus Canones [NALlinO, I, pág.135-138; III, pp. 203-208; BossONG, 1978, pp. 98-102]. El tratado está ampliamente ilustrado con diversos dibujos del reloj de sol para la latitud de Toledo según una nota escrita en el margen de uno de los folios del manuscrito [RICO, IV, p. 14]. Cabe decir que entre las figuras que acompañan al manuscrito, que ya se irán comentando detalladamente a medida que se vaya llegando a ellas, y los supuestos calcos de las mismas que se pueden ver en la edición de Rico y Sinobas [RICO, IV] hay algunas diferencias también dignas de mencionar en su momento. 
La obra astronómica alfonsí citada proporciona todos los conocimientos necesarios para poder construir y utilizar correctamente un reloj de sol horizontal. Su autor es Rabiçag (también llamado Rabbí Çag, Rabiçag Aben Cayut, Rabbi Isaac haHazzan ben Sid y Rabbi Issac ben Sid Hazzan de Toledo), uno de los tres colaboradores judíos de Alfonso X que, junto con Yehudá ben Moshé y Samuel ha-Levi, formaban el «grupo de Toledo». Dentro de la obra alfonsí, además de ser el traductor del Almanaque de Azarquiel y de los Canones de al-Battanì y el coautor de las Tablas Alfonsíes (1263-1272) con Yehudá ben Moshé, Rabiçag es autor de los siguientes Libros: del Quadrante (1277), del Astrolabio Llano, del Astrolabio Redondo, de la Lámina Universal, de las Armellas, dell Ataçir, del Relogio de la Piedra de la Sombra, del Relogio dell Agua, del Relogio dell Argent Vivo, del Palacio de las Horas y del quadrante Sennero. Tal y como se nos dice en el prólogo, el autor pretende escribir un tratado suficientemente completo que no necesite de ningún otro para poder construir el reloj solar. El libro está dividido en dos partes: una primera que consta de catorce capítulos donde se nos explica, paso a paso, todo lo necesario para la fabricación de la piedra y una segunda, dividida en cuatro capítulos, que nos aclara cómo utilizarla.

En los cinco primeros capítulos del libro tenemos expuestas una serie de reglas de cálculo de variables astronómicas necesarias para la construcción de las líneas del reloj de sol que, asimismo, son las reglas básicas de la mayoría de tratados astronómicos.

En primer lugar [RICO, IV, p. 5], hemos de conocer el arco diurno $d$ mediante la fórmula recogida ya en el Almagesto de Ptolomeo [II, 8; TOOMER, p. 99]

$$
\mathrm{d}=\alpha_{\varphi}\left(180^{\circ}+G\right)-\alpha_{\varphi}(G)
$$

donde $\alpha_{\varphi}(G)$ representa la ascensión oblicua del grado $G$ de la eclíptica. A partir de $d$ se calcula la duración de una hora temporal del día como su doceava parte.

Seguidamente nos enseña que, para saber la altura meridiana del Sol, hemos de conocer la colatitud del lugar y la declinación del Sol:

$$
h_{m}=\bar{\varphi} \pm \delta
$$

donde $\bar{\varphi}=90^{\circ}-\varphi$ es la colatitud y $\delta$ la declinación. El mismo cálculo se repite en el capítulo décimo sexto de los Cánones de al-Battānī [NALlinO, I, p. 30, III, pág. 44]; BOSSONG, 1978, p. 72] donde hay dos procedimientos equivalentes para calcular la altura meridiana del Sol.

En este capítulo tenemos también una tabla de declinaciones calculadas de grado en grado hasta $90^{\circ}$ y de la que se deduce el valor del ángulo entre eclíptica y ecuador $\varepsilon=23 ; 32,30^{\circ}$ [RICO, IV, p. 6], parámetro del que tenemos dos referencias más. La 
primera en el zî̀ de al-Sanŷufinì (París BN Arabe 6040), manual de astronomía mongol del siglo XIV [KENNEDY, 1987/88; COMES, 1989] que contiene una tabla de declinación solar para este valor de la oblicuidad de la eclíptica. La otra referencia es la tabla de declinaciones del zîy de Ibn Ishāq. Julio Samsó me documenta otra fuente titulada Nāta'îy al-afkār fi sărh rawdat al-azhār (c. 1514; ms. Cairo K4311 fol. 13r y ms. British Library Or. 411 fol. 27̀v). Según esta obra, Ibn Ishāq afirma que este parámetro lo estableció, por observación, un astrónomo de Meknes en el 602 H/ 12051206. El hecho es que la tabla del libro alfonsí es una copia de la de Ibn Ishāq ya que repite los mismos errores. De una tabla a otra sólo cabe mencionar cinco diferencias:

\begin{tabular}{|c|c|c|c|}
\hline & $\begin{array}{l}\text { Tabla de } \\
\text { Ibn Ishāq }\end{array}$ & $\begin{array}{c}\text { Tabla en } \\
\text { Alfonso X }\end{array}$ & $\begin{array}{c}\text { Valor } \\
\text { Recalculado }\end{array}$ \\
\hline 25 & $9 ; 43,5$ & $9 ; 43,6$ & $9 ; 43,5$ \\
\hline 40 & $14 ; 52,36$ & $14 ; 22,36$ & $14,52,36$ \\
\hline 57 & $19 ; 34,17$ & $19 ; 34,16$ & $19,34,16$ \\
\hline 63 & $20 ; 50,32$ & $20 ; 50,52$ & $20,50,51$ \\
\hline 70 & $22 ; 2,42$ & $22 ; 2,13$ & $22 ; 2,41$ \\
\hline
\end{tabular}

Las diferencias que corresponden a los argumentos $40^{\circ}, 57^{\circ}$ y $63^{\circ}$ son fáciles de explicar por confusiones en el sistema alfanumérico del $a b \hat{y} a d$.

En el cuarto capítulo [RICO, IV, p. 7] tenemos la regla para la determinación de la altura del Sol a partir de las horas de día transcurridas, que es exactamente la misma que nos da al-Battani en el capítulo decimoséptimo de sus Canones [NALLINO, I, p. 31, III, p. 46; BossoNG, 1978, pp. 56- 58]. La fórmula que hemos de seguir es:

$$
\operatorname{sen} h_{t}=\frac{(\operatorname{vers} d-\operatorname{vers} t) \cdot \operatorname{sen} h_{m}}{\operatorname{vers} d}
$$

donde $h_{t}=$ altura solar en un instante dado, $h_{m}=$ altura meridiana, $t=$ ángulo horario (longura yguada) y $d=$ arco semidiurno. El factor

vers $d$ - vers $t$

recibe el nombre de longura suelta en el Libro del relogio. Conviene señalar que Rabiçag, traductor de los Canones de al-Battānī, no utiliza siempre la misma terminología: la longura yguada se convierte en el arredramiento del Sol de mediel cielo en la traducción de los Cánones y la longura suelta pierde su nombre.

La fórmula es equivalente a la que encontramos en las Tablas Afonsíes [SAMSÓ, 1987]:

$$
\text { vers } t=\operatorname{vers} d-\frac{\operatorname{vers} d \cdot \operatorname{sen} h_{t}}{\operatorname{sen} h_{m}}
$$


Esta regla tiene antecedentes hindúes (Brahmagupta) y fue formulada por Habǎs al-Hāsib y probada por Abū al-Wafā' al-Buzyaānì y al-Bïrūnī [NADIR, 1960; KENṄEDY, 1973, KENNEDY, 1976].

En quinto lugar, el Libro del Relogio de la Piedra de la Sombra nos provee de una tabla de sombras espandidas (12 veces la cotangente) calculada para un gnomon igual a 12 partes [RICO, IV, p. 8]. Esta tabla [según AUSEJO, 1983] no proviene directamente ni de la tabla de al-Jwärizmi [SUTER, 1986, p. 672] ni de la de al-Battānī [NALLINO, II, p. 60] que, excepto alguna discrepancia nunca superior a 1" de diferencia, son las mismas. También según [AUSEJO, 1983], la primera parte de la misma, de $1^{\circ}$ a $45^{\circ}$, parece ser una mala copia de la tabla de al-Battānì mientras que el resto está calculado con la expresión $\frac{12 \cdot \operatorname{sen}(90-\alpha)}{\operatorname{sen} \alpha}$, a partir de los valores de la función seno de al-Battānī.

La regla que sigue es la del cálculo del azimut en función de la altura [RICO, IV, pp. 8-9]. La fórmula correspondiente es equivalente a:

$$
\operatorname{sen} \alpha=\frac{R \cdot(\operatorname{sen} r+\operatorname{sen} f)}{\cos \varphi}
$$

donde: $\alpha=$ azimut

$$
\begin{aligned}
& r=\text { anchura de oriente o amplitud ortiva y } \operatorname{sen} r=\frac{R \cdot \operatorname{sen} \delta}{\cos \varphi} \\
& f=\text { diuersidat del orizon y } \operatorname{sen} f=\frac{\operatorname{sen} h \cdot \operatorname{sen} \varphi}{\cos \varphi}=\operatorname{sen} h \cdot \tan \varphi
\end{aligned}
$$

Esta formulación ya la encontramos en los Canones de Albateni y también en alBïrūnī [KENNEDY, 1973; KENNEDY, 1976]. En al-Battānī, la regla viene dada en el capítulo undécimo de sus Cánones [NALLINO, I, p. 23 y III, pp. 33-34; BossONG, pp. 58-59] donde nos da la misma descripción con la misma terminología (anchura dell orientamiento y diuersidat dell orizon), que incluye el uso de la palabra cuerda por seno siguiendo lo que el propio al-Battānī ha dicho en el capítulo tercero:

\footnotetext{
«...y non por tornar una razón muchas ueces dezimos te que en todel logar que nos dixieremos en este libro cuerda: sabe que es la cuerda que es partida por medio...» [BOSSONG, p. 36; NALLINO, I, p. 10 y III, p. 15].
}

Esta analogía sin embargo, no se produce en el capítulo cuarto del Libro del Relogio [RICO, IV, p. 7] donde Rabiçag denomina a la función seno con el término signo. Del mismo modo en este texto, la función seno verso es denominada saeta mientras que en los Canones aparece la expresión cuerda retornada. 
Siguiendo esta tradición, el capítulo sexto del Libro del Relogio de la Piedra de la Sombra nos proporciona una tabla de cuerdas que, en realidad, es una tabla de senos de argumentos que van aumentando de grado en grado de $1^{\circ}$ a $90^{\circ}$ para un radio igual a 60 partes [RICO, IV, p. 10]. La tabla está obtenida a partir de la tabla de al-Jwārizmi [SUTER, pp. 169-170] o de la de al-Battānì [NALLINO, II, pp. 55-56] por redondeo de la segunda fracción sexagesimal [AUSEJO, 1983] ya que de los noventa valores tabulares sólo discrepa en tres ocasiones de la tabla redondeada y éstas se pueden atribuir a errores de copia. Ambas tablas orientales, como en el caso de la tabla de cotangentes, son básicamente la misma si exceptuamos que los argumentos de la primera aumentan de grado en grado mientras que los de la segunda lo hacen cada 30' y que, entre ambas, aparece ocasionalmente alguna diferencia del orden de 1".

Cambia ahora el tratado de tema para pasar a construir la piedra en sí [RICO, IV, p. 10]. La construcción alfonsí que ahora detallaré no discrepa mucho de la que se encuentra en los Canones (capítulo 56) [NALLINO, I, pp. 135-138 y 111, pp. 203-208; BossONG, 1978, pp. 98-102]. Lo primero que necesitamos para construir nuestra piedra de la sombra es una piedra bien lisa de forma rectangular tal que su anchura sea igual a dos tercios de su longitud. Con estas medidas Rabiçag recoge el consejo de al-Battānī quien nos invita a coger una piedra del tamaño que se quiera et lo meior que puedes fazer en esto; es que sea su anchura tanto commo los dos tercios de su longura. En ella se ha de dibujar la linna de anchura o linna de mediodia, recta que dividirá la piedra por la mitad de su longitud en dos partes iguales. Esta línea la hemos de dividir asimismo en tres segmentos iguales y por el extremo del primero de ellos, haremos pasar una recta perpendicular a la anterior a la que llamaremos linna de longura. Con el punto de interseccion de ambas líneas como centro, se traza una circunferencia cuyo radio sea menor que la sombra del principio de Cáncer, es decir:

$$
r<12 \cdot \cot (\bar{\varphi}+\varepsilon)
$$

que es la sombra más corta del año. Esta restricción para el radio no la encontramos en al-Battānī, quien nos da libertad absoluta en su medida. En los Canones todo este procedimiento es el mismo pero hecho a la inversa: primero se dibuja la circunferencia con centro en el mismo punto que en nuestra piedra y, posteriormente, se dibujan unas líneas que quadrean el cerco y que se cortan sobre dos angulos derechos, ya que no les llama líneas de longura y anchura. A pesar de esto, posteriormente en el capítulo de los Canones encontramos el término linna de mediel dia. Así, para la linna de longura, en el Libro del Relogio, Rabiçag evita tener que ir llamándole como en los Canones: la linna que es entre oriente y occidente. Este capítulo viene ilustrado con un dibujo esquemático de la piedra después de trazar en ella las líneas necesarias. En el manuscrito alfonsí, la línea de mediodía muestra una casi imperceptible curva justo en la intersección con la linna de longura. Además, esta 


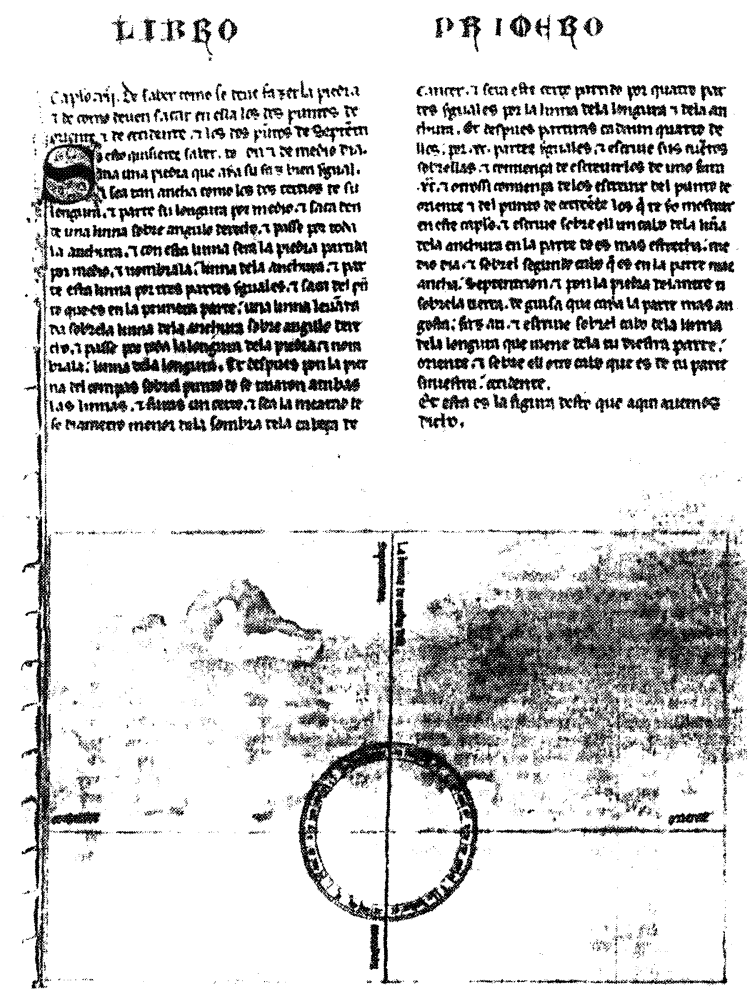

Figura 2.1. Manuscrito Villamil 156.

Biblioteca de la Universidad Complutense.

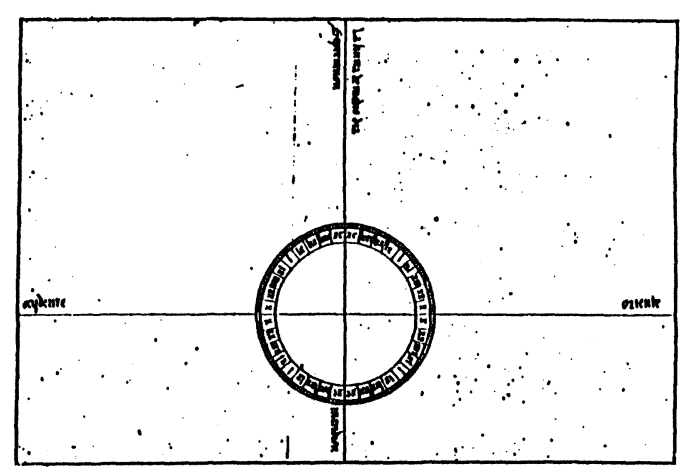

Figura 2.2. [Rico, IV, pág. 11] 
intersección no es perpendicular tal y como debería ser (figura 2.1). La edición de Rico y Sinobas reproduce bastante bien la piedra (figura 2.2) [RICO, IV, p. 11]. Tal y como hemos dibujado la circunferencia, ésta nos quedará partida en cuatro sectores iguales. Cada uno de estos cuadrantes se ha de dividir en noventa partes, de manera que nos quedará una circunferencia dividida en sus $360^{\circ}$. En cada cuadrante tomaremos el origen para la medida de los ángulos en los puntos comunes de la circunferencia y la línea de longura. El resultado es el que se puede ver en la figura 2.1.

Una vez hecho esto, hemos de dibujar en la piedra las líneas horarias y las líneas que corresponden al recorrido del extremo de la sombra del demostrador (gnomon) para los días de solsticio y de equinoccio. Estas líneas son secciones cónicas en todos los casos aunque varían según la latitud en la que nos encontremos:

- elipses, si $|\varphi|>90^{\circ}-\varepsilon$;

- parábolas, si $|\varphi|=90^{\circ}-\varepsilon$;

- hiperbolas, si $|\varphi|<90^{\circ}-\varepsilon$

En nuestro caso, estas líneas serán hipérbolas. Para llegar a ellas, en primer lugar [RICO, IV, p. 13] buscaremos los azimuts de las horas del principio de Capricornio siguiendo la regla dada en el capítulo sexto. Por simetría, sólo tendremos que buscar los azimuts de las cinco primeras horas ya que el de la sexta es $90^{\circ}$ y cae sobre la línea de mediodía. A partir de la séptima tenemos:

$$
\alpha\left(h_{\mathrm{k}}\right)=180-\alpha\left(h_{12-\mathrm{k}}\right), \quad \text { para } k=7,8,9,10,11 .
$$

donde $h_{k}$ es la altura del Sol después de haber transcurrido $k$ horas de día, o bien, si tenemos en cuenta la división que se ha hecho sobre la circunferencia que tenemos en la piedra:

$$
\alpha\left(h_{k}\right)=\alpha\left(h_{12-k}\right), \quad \text { para } k=7,8,9,10,11 .
$$

donde si $\alpha\left(h_{12-k}\right)$ está en el segundo cuadrante, entonces, $\alpha\left(h_{k}\right)$ está en el primero. Los valores obtenidos los señalaremos sobre sus correspondientes graduaciones en la circunferencia que tenemos dibujada en la piedra.

Una vez hallados los azimuts de las horas de Capricornio y también los de las horas del principio de Cáncer por razonamiento análogo, hemos de buscar los extermos de sus sombras espandidas tal y como se ha explicado en el capítulo quinto del libro. Para marcar en la piedra las señales de las sombras, nos ayudaremos de una regla (llamada regla de la sombra). Esta regla [RICO, IV, p. 12] tendrá una longitud no menor que la sombra del principio de Capricornio. Estará graduada en función de la longitud del gnomon, el cual estará dividido en doce partes. Sacamos pues el valor de 
la sombra espandida de la primera hora temporal del principio de Capricornio y hacemos una señal en la regla en la graduación que corresponde a la sombra obtenida. Lo mismo hacemos para las siguientes horas teniendo en cuenta que tenemos simetría respecto de la sexta hora:

$$
s_{e}\left(h_{12-k}\right)=s_{e}\left(h_{k}\right), \text { para } k=7,8,9,10,11 .
$$

donde $s_{e}$ representa la función sombra espandida.

Ahora, ponemos la regla en la dirección de la sombra de la primera hora temporal, esto es, en la dirección que viene dada por el centro de la circunferencia inicial y el azimut de la primera hora, y marcamos sobre la piedra en el lugar que indique la señal que hemos hecho en la regla para la primera hora temporal (figura 3).

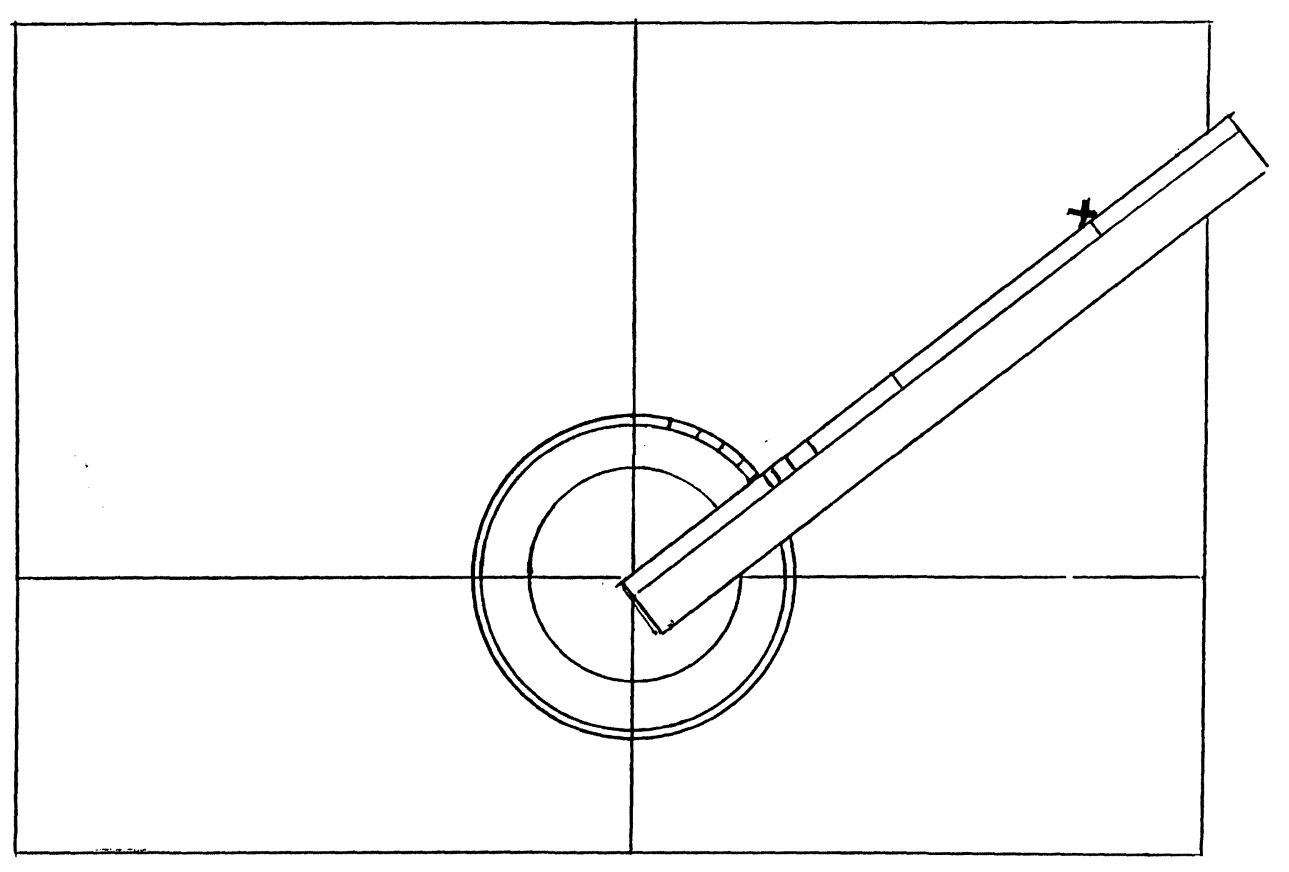

Figura 3. 
Este proceso lo repetimos para las restantes once horas del día. La figura 4 muestra el resultado obtenido hasta el momento.

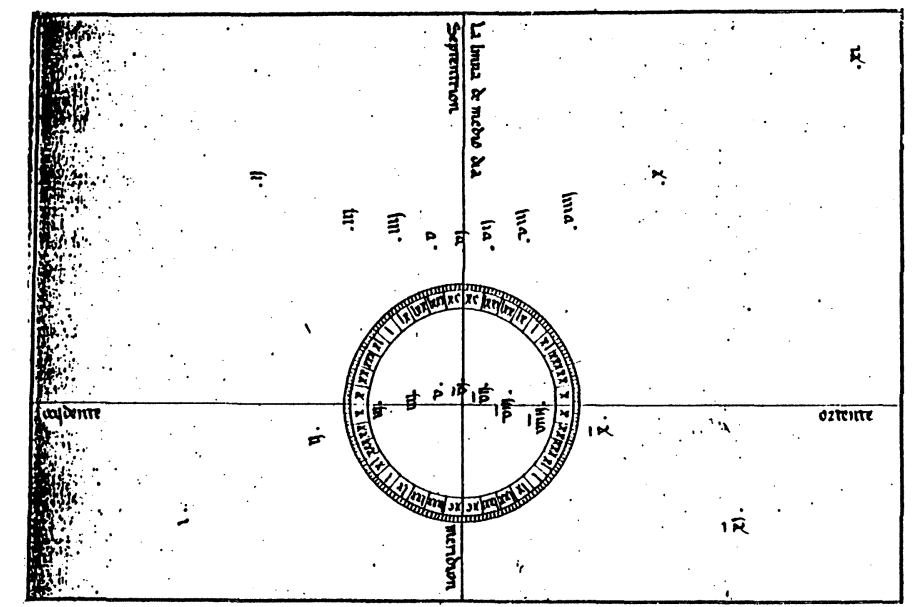

Figura 4. [RIco, IV, pág. 15]

Del capítulo VII al X (desde el trazado de las primeras líneas en la piedra hasta la figura 4), Rabiçag consigue de manera clara esquematizar el procedimiento que da al-Battānì. En los Canones, después de invitarnos a saber las sombras y los azimuts de las distintas horas de los principios de Cáncer y Capricomio, también hemos de coger una regla que sea bien egual, de longitud mayor o igual que la sombra de la primera hora de Capricornio e igualmente graduada que la regla de la Sombra alfonsí. La utilidad de esta regla es la misma en ambas obras con lo que, finalmente, el resultado obtenido es también el mismo.

Trazaremos ahora las líneas horarias del reloj [RICO, IV, p. 15]. La línea horaria de la primera hora temporal la dibujamos uniendo la señal de la primera hora de Capricornio con la de la primera hora de Cáncer, y lo mismo para todas las demás horas. Hemos de unir también todas las señales de las horas de Capricornio por un lado y por otro, todas las de Cáncer. Ambas líneas son dos hipérbolas. El autor de este tratado nos explica que para dibujar estas secciones cónicas basta con dibujar un arco de circunferencia que una las señales cercanas a la línea del mediodía y unir las demás con líneas rectas. Esta identificación que se hace entre una hipérbola y un arco de circunferencia prolongado con dos rectas no lo encontramos en los Canones, donde al-Battānỉ nos invita a hacer la unión mediante simples segmentos rectos. Tampoco la encontramos en la propia ilustración que acompaña al texto alfonsí donde las 
señales de las horas están unidas también mediante segmentos rectos. En la tradición andalusí las uniones entre los puntos han sido trazadas mediante líneas rectas en dos de los ocho cuadrantes conservados: en otros dos casos (cuadrante de Córdoba y segundo de los cuadrantes de Medina Azahara en la ordenación de [KING, 1992]) puede haberse utilizado un procedimiento similar al descrito en el texto alfonsí, mientras que en el cuadrante de Granada se ha utilizado un arco de circunferencia. En los otros tres cuadrantes, los restos que tenemos de ellos no nos permiten asegurar nada [fotografías en KING, 1992].

Una demostración rigurosa de dicha identificación puede ser hecha a partir del centro y del radio de curvatura de una hipérbola. En una curva cualquiera, podemos considerar la circunferencia tangente a un punto dado por su lado cóncavo de manera que no podamos trazar ninguna otra circunferencia tangente al punto entre la primera y la curva (figura 5). Al radio de esta circunferencia lo llamaremos radio de curvatura de la curva en el punto y a su centro, centro de curvatura.

Para el caso de la hipérbola, teniendo en cuenta que con transformaciones adecuadas nos podemos reducir al caso de una hipérbola equilátera que nos viene dada por la expresión:

$$
x^{2}-y^{2}=1
$$

su radio de curvatura en un punto $(a, b)$ dado es: $R=\left(a^{2}+b^{2}\right)^{\frac{3}{2}}$ y su centro de curvatura es el punto: $\left(2 a^{3},-2 b^{3}\right)$.

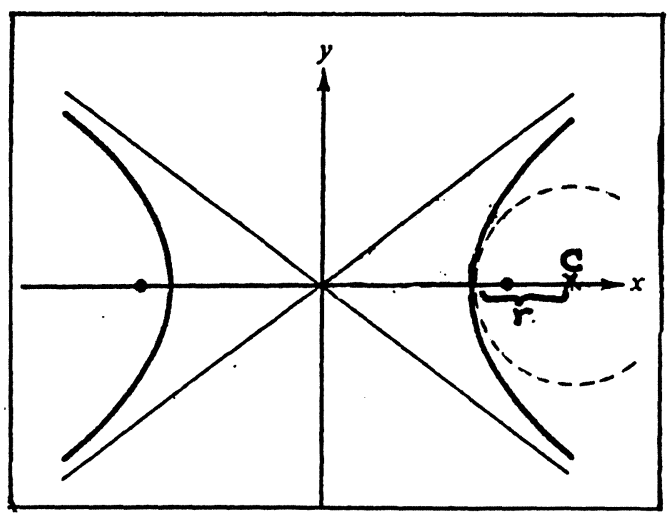

Figura 5. Centro y radio curvatura de una hipérbola.

Lo que se pretende demostrar es que para los valores del intervalo $[1,1+\xi]$ para $\xi$ positivo suficientemente pequeño, el arco de hipérbola es muy similar a un arco de 
circunferencia y para valores de la abscisa suficientemente grandes, es similar a una recta. Traducido a términos de centros y radios de curvatura, hay que ver que, en el primer caso, el radio y el centro de curvatura de los puntos $x \in[1,1+\xi]$ son casi los mismos en todos los casos y, en el segundo, basta con ver que el radio de curvatura se hace infinito. En efecto:

$$
\begin{aligned}
& R(x=1)=1 \quad \text { y } \quad R(x=1+\xi)=\left(2(1+\xi)^{2}-1\right)^{\frac{3}{2}} \cong 1^{\frac{3}{2}}=1 \text { ya que } \xi \ll 1 \\
& C(x=1)=(2,0) \text { y } C(x=1+\xi) \cong(2,0)
\end{aligned}
$$

y para el segundo caso:

$$
\lim _{x \rightarrow \infty} R(x)=\lim _{x \rightarrow \infty}\left(x^{2}+\left(\sqrt{x^{2}-1}\right)^{2}\right)^{\frac{3}{2}}=\lim _{x \rightarrow \infty}\left(2 x^{2}-1\right)^{\frac{3}{2}}=\infty
$$

Volviendo al reloj de sol, una vez unidas todas las señales hechas sobre la piedra, ésta pasa a tener un aspecto similar al que se puede ver en las figuras 6.1 y 6.2. El dibujo que encontramos en el manuscrito alfonsí original (figura 6.1) no muestra el cambio de una hipérbola por la circunferencia y las rectas; las uniones de las señales están hechas con segmentos pero con una particularidad: no todos los segmentos tienen su origen en un punto y su final en el inmediatamente posterior sino que hay algunos que unen puntos que no son correlativos. Así, para Capricornio, los puntos extremos de los segmentos son las horas primera, tercera, sexta, séptima, octava, novena, décima y undécima y también, quizá, la cuarta, y para Cáncer, la quinta y, quizá, la octava son las únicas que no lo son. En la edición de Rico y Sinobas (figura 6.2)

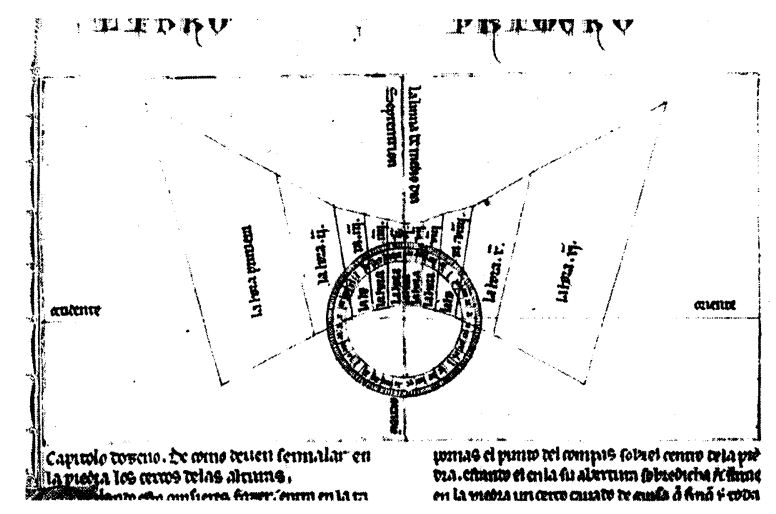

Figura 6.1. Manuscrito Villamil 156

Biblioteca de la Universidad Complutense 


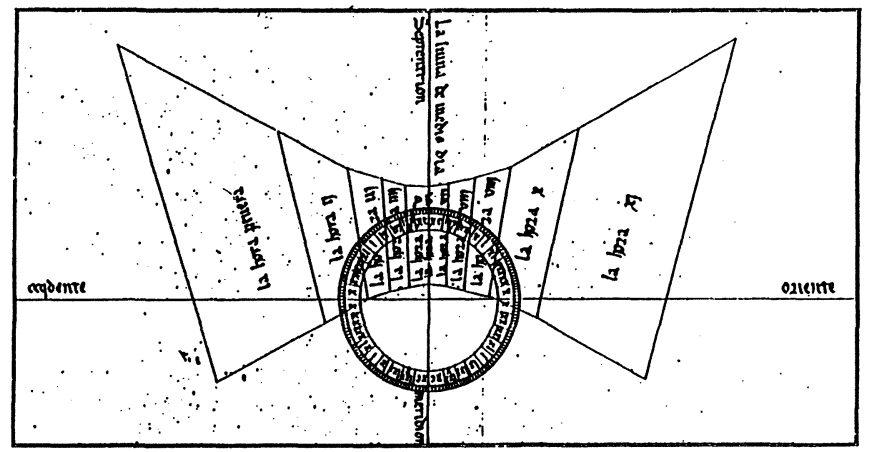

Figura 6.2 [RICO, IV, pág. 16].

[RICO, IV, p. 16] no se encuentran las mismas uniones, teniendo para Capricornio como puntos extremos de las mismas, las horas primera, tercera, quinta, sexta, séptima, octava, novena y undécima mientras que para Cáncer, las horas segunda, quinta y octava son las que no son extremo de ninguna unión. En ambos dibujos, tanto el original como la reproducción, las medidas de la piedra han dejado de tener la proporción que Rabiçag en un principio sugería (anchura $=2 / 3$ de la longitud) para pasar a tener, como en muchos de los dibujos del Libro del relogio, unas medidas donde, de largo, la piedra mide alrededor de dos veces lo que mide de ancho.

Nos queda ahora dibujar en la piedra las circunferencias que nos sirven para reconocer la altura del Sol en un momento dado [RICO, IV, p. 16]. Para dibujarlas, hay que entrar en la tabla de sombras espandidas con el valor de la altura que queramos tener identificado y obtener su sombra. Después, tomando como referencia la partición hecha en la regla de la sombra, abrimos el compás tanto como los distintos valores que obtenemos para las sombras y vamos dibujando las circunferencias con dichos radios y centro en el centro de la piedra, escribiendo en algún lugar visible la leyenda de su altura correspondiente (figuras 7.1 y 7.2). Estos círculos de las alturas son una innovación con respecto a los ocho cuadrantes andalusíes conservados y con respecto al reloj de sol de los Canones. Aquí, los dibujos que acompañan al texto también muestran diferencias y ya no sólo entre sí, sino que también son algo distintos de los que se han descrito anteriormente. En el manuscrito original (figura 7.1) tenemos, con respecto a la descripción anterior, que las horas segunda, quinta y décima de Capricornio son extremo de unión mientras que la séptima hora deja de serlo. Por otro lado, las horas de Cáncer están unidas una a una. En la edición de Rico y Sinobas (figura 7.2) [RICO, IV, p. 17] sólo hay, tanto para Capricomio como para Cáncer, una hora que no es extremo de ninguna unión y es, respectivamente, la décima y la octava. 


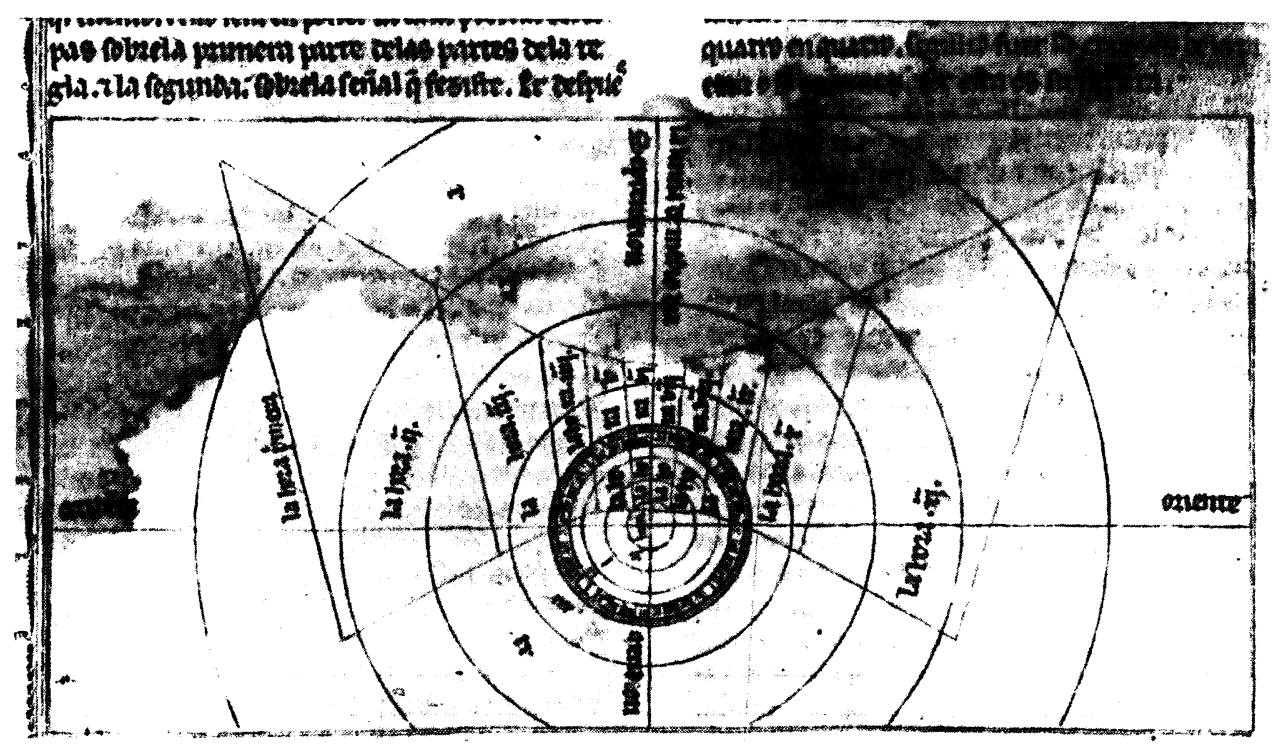

Figura 7.1 Manuscrito Villamil 156. Biblioteca de la Universidad Complutense.

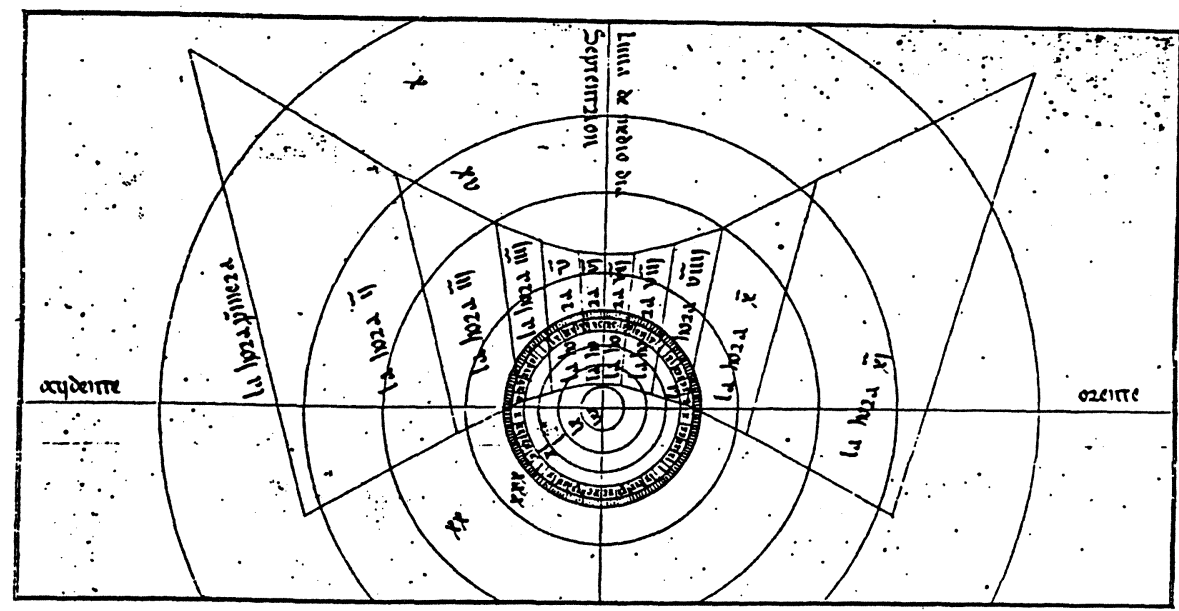

Figura 7.2. [RICO, IV, pág. 17]

Para acabar con la construcción de la piedra de la sombra, sólo falta construir el gnomon (demostrador) y colocar la piedra correctamente [RICO, IV, pp. 18-19]. El 180 
gnomon, como ya se ha dicho, está dividido en doce partes a las que hay que añadir una prolongación equivalente al grosor de la piedra con objeto de asentarlo en la misma. Asimismo, ha de terminar en punta con el fin de no distorsionar la lectura de la hora sobre el reloj. En el centro de todas las circunferencias anteriores hacemos un agujero, de modo que allí va a estar clavado el gnomon. Rabiçag explica cómo ha de ser aproximadamente la forma del gnomon de modo que su anchura es tres tanto del diámetro del forado en el centro del çerco que es en la piedra. En el torno, el gnomon será moldeado de manera que la prolongación de las doce partes de las que consta su longitud tendrá forma cilíndrica de diámetro igual a un tercio del diámetro de la base del gnomon de la que sobresale. Esta explicación se la ahorra al-Battāni, quien de su cathete sólo dice que ha de estar metido en el torno y que sea de arambre o de fierro redondo. Rabiçag además de añadir toda la explicación anterior, ofrece la posibilidad de que el gnomon sea de qual metal quissieres. El gnomon es entonces colocado perpendicularmente a la piedra en el centro de la misma. Aquí también Rabiçag añade al hecho que el cathete de al-Battāni se non mezca; nin se tuelga de so logar, sinon que finque sobre angulos derechos [BOSSONG, p. 100; NALLINO, I, p. 136, y III. p. 204]. Añade también una comprobación de la perpendicularidad mediante la igualdad entre las longitudes entre el extremo del gnomon y la intersección de la base del mismo con las líneas de longura y anchura.

Finalmente, para armar la piedra, se ha de tomar la altura del Sol al comienzo de una hora del día tal y como se ha dicho en el capítulo cuarto y sacar su azimut de acuerdo con el capítulo sexto. Ponemos la regla sobre el centro de la piedra y sobre el azimut encontrado y hacemos una señal, que podamos borrar después, sobre el extremo de la sombra correspondiente. Colocamos la piedra en el suelo o en una superficie paralela al horizonte. En el momento que la sombra espandida marcada sobre la piedra sea la misma que la encontrada al principio de esta operación, haremos coincidir la sombra del gnomon con el azimut encontrado también al principio, de tal modo que tendremos así la piedra correctamente armada. En estos momentos es cuando se puede fijar la piedra a la superficie. Esta manera de orientar la piedra también la recoge al-Battānĩ quien, después de dar el método del «círculo indio» para la determinación de la línea meridiana, ofrece esta segunda posibilidad. Quizá Rabiçag sólo propone este segundo método a propósito de los círculos para medir las alturas que ha dibujado en la piedra y que en los Canones no se mencionan. Además, alBattāni tiene un problema: una vez sabida la altura de una de las horas del día y su azimut, podría pasar que la longitud de la sombra fuese menor que el radio de la circunferencia donde se han de marcar los azimuts, de manera que el autor requiere para este posible caso el uso de un filo delgado que esté señalando el azimut correspondiente desde el centro de la piedra. Este detalle no le puede suceder a Rabiçag, ya que recordemos que cuando ha trazado la circunferencia ha puesto la condición de que su radio sea menor que la sombra más corta del año. Ahora, por lo tanto, se entiende esta restricción que, en su momento, parecía innecesaria. 
La segunda parte del tratado [RICO, IV, pp. 21-23] explica cómo utilizar la piedra una vez armada y se divide en cuatro cortos capítulos. En el primero nos explica cómo saber el número de horas temporales transcurridas del día: sólo hay que ver sobre qué línea horaria se encuentra el extremo de la sombra del demostrador. Si queremos saber el número de horas equinocciales transcurridas, se aplica la regla equivalente a:

$$
H=\frac{h \cdot d}{15 \cdot 12}
$$

donde $d=$ arco diurno, $h=$ número de horas temporales pasadas y $H=$ número de horas equinocciales transcurridas.

El segundo capítulo nos dice que, para saber la altura del Sol en cualquier hora del día, se ha de mirar sobre qué circunferencia de las que marcan las alturas se encuentra el extremo de la sombra del demostrador y la leyenda que marque: ésa será la altura que buscamos. Además, si no está exactamente sobre la circunferencia, interpolaremos linealmente.

Sabiendo el valor de la altura del Sol, podemos saber el valor de su sombra espandida entrando en la tabla del quinto capítulo. Si lo que se quiere saber es la sombra conversa (doce veces la tangente), hay que entrar en la tabla con el ángulo complementario a la altura que tenemos, es decir:

$$
s_{c}=12 \cdot \cot \left(90^{\circ}-h\right)=12 \cdot \tan h
$$

El último capítulo nos dice cómo saber el azimut de cualquier hora del día mirando el arco que señale la sombra del demostrador sobre la circunferencia graduada que tenemos en la piedra.

Así acaba el Libro del Relogio dicho de la Piedra de la Sombra, tratado en el que nos dice cómo construir un reloj de sol y que no discrepa en casi nada de la construcción dada por al-Battānī en sus Canones. Incluso si nos fijamos en las tablas, excepto la tabla de declinaciones de al-Battānī en que la oblicuidad de la eclíptica es $\varepsilon=$ $23 ; 35^{\circ}$ por los $\varepsilon=23 ; 32,30^{\circ}$ de aquí, las otras dos, la de senos y la de cotangentes beben de la fuente oriental. Por lo tanto, se puede afirmar que el tratado alfonsí es una buena reconstrucción del reloj de sol de al-Battānī y que está muy influido por sus tablas. Hay que tener en cuenta que Rabiçag debía conocer bien los Canones de al-Battānī, ya que fue él quien los tradujo del árabe al castellano [MILLÁs, 1956; ROMANO, 1991]. Por lo que se refiere a la tabla de declinaciones, está claro que es una copia de la tabla de Ibn Ishāq. Se podría por lo tanto resumir que, teniendo que escribir un tratado sobre la construcción de un reloj de sol, Rabiçag, quien ya había traducido al castellano los Canones de al-Battānī, vio en ellos el procedimiento correcto a seguir para la construcción del instrumento. Siguió la construcción de al- 
Battānĩ añadiendo a ella detalles y comprobaciones que no contenía, como los círculos para determinar la altura del Sol. Estas adiciones facilitan bastante la construcción además de poner en orden muchos conceptos que en los Canones encontramos mezclados con más reglas y conceptos astronómicos. Así, el Libro del Relogio dicho de la Piedra de la Sombra constituye una mejora respecto al texto de al-Battāni en lo que se refiere a la construcción del reloj de sol.

\section{AGRADECIMIENTOS}

Este trabajo ha sido realizado en el marco del proyecto de investigación «Astronomía teórica y tablas astronómicas en al-Andalus y el Magrib en los siglos XII-XIV» subvencionado por la DGICYT.

\section{BIBLIOGRAFÍA}

AUSEJO, E. (1983), «Sobre los conocimientos trigonométricos en los Libros del Saber de Astronomía de Alfonso X el Sabio». Llull, vol. 6, pp. 5-36.

BARCELÓ, C., y LABARTA, A. (1988), «Ocho relojes de sol hispano-musulmanes», AlQantara, IX, pp. 231-247.

Bossong, G. (1978), Los Cánones de Albateni. Max Niemeyer Verlag. Tubingen.

Cabanelas, D. (1958), «Relojes de sol hispano-musulmanes». Al-Andalus XXIII, pp. 391-406.

CASULlERAS, J. (1993), «Descripciones de un cuadrante solar atípico en el occidente musulmạ́n», Al-Qantara XIV,1, pp. 65-75.

CASULLERAS, J. (1996), «El último capítulo del Kitāb al-Asrār fi natā 'iŷ al-afkār». En J. CASUlleras y J. SAMSó (eds.), From Baghdad to Barcelona, Vol. II, Barcelona, pp. 613-654.

Comes, M. (1989), «À propos de l'influence d'al-Zarqālluh en Afrique du Nord: L'apogée solaire et l'obliquité de l'écliptique dans le zỉdj d'Ibn Isḥăq», Actas del II Coloquio HispanoMarroqui de Ciencias Históricas, pp. 147-159, Madrid.

DAVIDIAN, M.-L. (1983), «Al-Birūnī on the time of day from shadow lengths». E. S. KenNEDY, Colleagues and Former Students, Studies in the Islamic Exact Sciences, pp. 274279, Beirut.

EsteBAN, E., y Ros, R. M. (1996), «Líneas de calendario en un reloj de solar para diferentes lugares de la Tierra», Universo, julio-agosto, pp. 88-91.

IBN AL-RAQQĀM AL-ANDALUSí (1988), Risāla fi ${ }^{\circ}$ ilm al-zulāl, editado, traducido y comentado por Joan Carandell, Barcelona.

KENNEDY, E. S. (1973), A Commentary upon Bīrūnī's Kitäb Tahdid al-Amākin, American University of Beirut, Beirut.

KENNEDY, E. S. (1976), The Exhaustive Treatise on Shadows by Abū al-Rayhān Muhammad b. Ahmad al-Bīrūnī, Translation and Commentary (2 vols), IHAS, Aleppo. 


\section{CARLOS DORCE POLO}

KENNEDY, E. S. (1987/88), «Eclipse Predictions in Arabic Astronomical Tables prepared for the Mongol Viceroy of Tibet», Zeitschrift für Geschichte der Arabisch-Islamischen Wissenschaften, Band 4 (1987/88).

KING, D. (1978), «Three Sundials from Islamic Andalusia», Journal for the History of Arabic Science II, 2, pp. 358-392.

KING, D. (1992), «Los Cuadrantes Solares Andalusíes». J. VeRneT y J. SAMSÓ (eds.), El Legado Científico Andalusí, pp. 89-102, Madrid, 1992.

LABARTA, A., y BARCELÓ, C. (1995), «Un nuevo fragmento de reloj de sol andalusí», AlQantara, XVI, 1, pp. 147-150.

Millás VAllicrosa, J. M. (1956), «Una nueva obra astronómica alfonsí: El tratado del cuadrante 'sennero'», Al-Andalus, XXI, pp. 59-92.

NADIR, N (1960), «Abū al-Wafa'' on the Solar Altitude», The Mathematical Teacher 53, pp. 460-463. Reprinted in E. S. KENNEDY et al. (1983), Studies in the Islamic Exact Sciences, pp. 280-283, Beirut.

Nallino, C. A. (1969), Al-Battānī Opus Astronomicum (3 vols.), Mediolani Insubrum, 1899, 1903, 1907. Reprint Minerva GMBH. Unveranderten Nachdruck, Franckfurt.

Procter, E. (1951), Alfonso X of Castile, patron of literature and learning, Oxford at the Clarendon Press.

Rico y Sinobas, M. (1866), Libros del Saber de Astronomía del rey D. Alfonso X de Castilla, Volumen IV, Madrid.

Romano, D. (1991), «Le opere scientifiche di Alfonso X e l'intervento degli ebrei», De Historia Judía Hispánica, Col.lecció Homenatges, Universitat de Barcelona Publicacions, Barcelona, pp. 147-181.

Roth, N., «Jewish Translators at the Court of Alfonso X», Thought, Vol. 60, N²39, diciembre 1985, pp. 439-455.

SAMsó, J. (1987), «Alfonso X and Arabic Astronomy». M. COMES, R. PUIG y J. SAMSó (eds.), De Astronomia Alphonsi Regis, pp. 23-38, Barcelona.

SAMSÓ, J. (1992), Las ciencias de los antiguos en Al-Andalus, Colecciones Al-Andalus, Mapfre, Madrid.

SÁNCHEZ PÉReZ, J. A. (1955), La Personalidad Cientifica y los relojes de Alfonso X el Sabio, Publicaciones de la Academia Alfonso X el Sabio, Murcia.

SUTER, H. (1914), Die Astronomischen Tafeln des Muhammed ibn Mūsā al-Khwārizmì in der Bearteitung des Maslama ibn Ahmed al-Madjrịtī und der latein. Uebersetzung des Athelhard von Bath, Copenhague. Reprint: SUTER, H. (1986), Beiträge zur Geschichte der Mathematik und Astronomie im Islam, I, Franckfurt am Main.

TOOMER, G. J. Ptolemy's Almagest. Duckworth, London 1984. 